\title{
Overview of magnetic resonance lymphography for imaging lymphoedema
}

\author{
Alyssa Calderwood ${ }^{1}$, Belinda Thompson ${ }^{2}$, Kevin Ho-Shon $^{2}$, Hiroo Suami ${ }^{2}$ \\ 'Department of General Surgery, Fiona Stanley Hospital, Murdoch, WA 6150, Australia. \\ ${ }^{2}$ Faculty of Medicine, Health and Human Sciences, Macquarie University, Sydney, NSW 2109, Australia.
}

Correspondence to: Prof. Hiroo Suami, Faculty of Medicine, Health and Human Sciences, Level 1, 75 Talavera Rd, Macquarie University, 2109 NSW, Australia. E-mail: hiroo.suami@mq.edu.au

How to cite this article: Calderwood A, Thompson B, Ho-Shon K, Suami H. Overview of magnetic resonance lymphography for imaging lymphoedema. Plast Aesthet Res 2021;8:40. https://dx.doi.org/10.20517/2347-9264.2021.14

Received: 23 Feb 2021 First Decision: 6 Apr 2021 Revised: 11 Jun 2021 Accepted: 28 Jun 2021 First online: 5 Jul 2021

Academic Editors: Matthew L lorio, Isao Koshima Copy Editor: Xi-Jun Chen Production Editor: Xi-Jun Chen

\begin{abstract}
Lymphoedema is a chronic and debilitating condition commonly caused by cancer therapies, including lymph node dissection and radiotherapy in developed countries. A range of imaging modalities is used to view the lymphatic system for proper diagnosis, staging, and management of lymphoedema. Lymphoscintigraphy is the current gold standard imaging modality of the lymphatic system. However, magnetic resonance lymphography (MRL) is showing potential benefits in lymphoedema assessment and surgical planning. A literature review was compiled from published articles, incorporating their background literature, research outcomes, and recommendations to review the technique, application, and limitations of MRL. MRL is minimally invasive with no ionizing radiation, providing both functional and anatomical details of the lymphatic system with a higher spatial resolution than conventional lymphoscintigraphy. It shows promising results in the staging, surgical work-up, and surveillance for individuals with both primary and secondary lymphoedema.
\end{abstract}

Keywords: Magnetic resonance lymphography, lymphangiography, lymphoedema, lymphatic system, imaging

\section{INTRODUCTION}

Lymphoedema is a chronic and debilitating condition affecting between 90-250 million people worldwide, most commonly caused by malignancy and its treatment in developed countries ${ }^{[1-10]}$. It is characterised by 
the retention of protein-rich fluid in the interstitial tissue due to a disruption of lymph flow through the lymphatic system. It eventually results in limb deformity, skin changes, impaired immune function, and skin infections ${ }^{[3,8,9,11]}$, which has physical and psychological impacts on patients. In the early stages of lymphoedema, protein-rich fluid collects in the subcutaneous tissue, resulting in pitting oedema. It is later replaced by the accumulation of adipose tissue and fibro-sclerotic tissue, which does not cause pitting but causes a "woody" feel to the affected limb $b^{[7,12]}$.

The initial management of lymphoedema is conservative; however, advances within this field have led to a range of surgical options. These include vascularized lymph node transfer, lymphovenous anastomosis (LVA), and liposuction for advanced disease. Optimising imaging techniques allows clinicians to evaluate the characteristics of a person's condition, which in turn directs clinical decisions on appropriate management. Conventional imaging of the lymphatic system has been radionuclide lymphoscintigraphy, which was introduced in the $1950 \mathrm{~S}^{[13]}$. However, lymphoscintigraphy can only provide low-resolution images without anatomical body landmarks. A relatively new imaging technique called magnetic resonance lymphangiography (MRL) has been developed to map the lymphatic system, identify the structural change of the lymphatics in lymphoedema and assist with pre-operative planning ${ }^{[2,4]}$. A combination of $\mathrm{T} 2$ weighted non-contrast MRL and contrast-enhanced T1-weighted MRL allows optimal visualisation of individual lymphatic vessels, lymph nodes, and areas of dermal backflow and provides both anatomical and functional assessments. This manuscript will discuss the current types of MRL sequences and the benefits and limitations of their use in lymphoedema.

\section{METHOD AND DATA COLLECTION}

A selection of published journal articles about the clinical use of MRL in upper and lower limb lymphoedema were collected from electronic databases, PubMed, Ovid, and CINAHL, to create a literature review. Search topics included "magnetic resonance lymphography", "magnetic resonance lymphangiography", "contrast MRL", "non-contrast MRL", and other related words. The research outcomes, recommendations, and discussion points of these articles were compared and summarised to create a review of the role of MRL in a clinical setting. A total of 35 articles were selected, dated from 2005 to 2020. These articles included a range of studies and literature review papers, with patient cohorts consisting of both primary and secondary lymphoedema in the upper or lower limbs. Only papers written in English were included.

\section{TYPES AND TECHNIQUES OF MRL}

MRL is a modification of $3 \mathrm{D}$ volumetric MR angiography ${ }^{[2]}$. There are three types of MRL sequences used in MRL.

\section{Patient position}

Patient position depends on the area being studied. With lower limb imaging, the patient is supine with their feet first in the MRI machine, whilst with upper limb imaging, the patient is prone, head first with their arms extended and palms down $\mathrm{n}^{[1-3,6,7,14-16]}$. This positioning allows contrast administration during the $\operatorname{scan}^{[2,6]}$.

\section{Image sequences}

As outlined below, multiple MRI sequences can be used to produce an optimal assessment, and all three are performed in the following sequence. First, a non-contrast heavily T2-weighted sequence is used to define the severity of the lymphoedema (non-contrast $M R L$ ). Then an enhanced high-resolution fat-suppressed T1-weighted sequence is performed at different time intervals following subcutaneous or intradermal 
contrast administration to visualise the individual lymphatic channels (contrast-enhanced $M R L$ ). Finally, a delayed sequence is performed with intravenous contrast injection to define the veins (venogram). Fast spinecho is used to obtain images in a shorter time to eliminate motion artefact ${ }^{[0,11,14,15,17-21]}$, and maximum intensity projection is commonly used to present the reconstructed images ${ }^{[1-4,6-8,11,14-19,21-28]}$.

\section{Contrast-enhanced MRL}

Contrast-enhanced MRL sequences provide not only anatomical information of the lymphatic channels and lymph nodes (to a higher resolution than non-contrast MRL and lymphoscintigraphy) but also information on the drainage patterns of the lymphatic system ${ }^{[7,8,14,21,27]}$. A paramagnetic macromolecule contrast agent is injected either subcutaneously or intradermally in the web spaces of the hand or foot, which is taken up into the lymphatic vessels and allows them to be visualised with high resolution fat-suppressed T1-weighted sequences ${ }^{[2,6,13,15,19,24,25,27,29]}$. Imaging acquisition is performed at either $5 \mathrm{~min}$ or $10 \mathrm{~min}$ intervals following contrast administration ${ }^{[2,6,7,14-16,19,26]}$. The lymphatics can be seen approximately 7 to 15 min after contrast injection $^{[26,29]}$. However, they tend to show better enhancement in the later phase (35-55 min after injection ${ }^{[1,266]}$. The size of the paramagnetic macromolecules is key to their preferential uptake in the lymphatic system and should be larger than $6-10 \mathrm{~nm}^{[21]}$.

The most common agent used in the included articles was gadopentetate dimeglumine, although there are currently 9 gadolinium-based contrast agents (GBCAs) commercially available ${ }^{[2]}$. These gadolinium-based agents are water-soluble, not subject to metabolism, and are excreted unchanged via the kidneys, therefore causing minor tissue damage, and are widely used with very few adverse effects reported in patients with normal renal function ${ }^{[2,3,14,16,18,23]}$. Gadopentetate dimeglumine was also reported to have benefits including its thermal stability, high relaxivity, and potential for weak protein binding ${ }^{[6]}$. However, it is noted that moving forward from 2019, linear contrast agents, such as gadopentate dimeglumine, are no longer used due to reports of gadolinium deposition in organs. Therefore, macrocyclic agents should be used from this point onwards.

Local anaesthetic is commonly mixed with the contrast to reduce pain at the injection site ${ }^{[1-3,7,15,1,18,24,30]}$. Although there is no consensus between the articles for which method of contrast administration is preferred, the subcutaneous injection may result in more contrast uptake in blood vessels rather than lymphatic vessels ${ }^{[7,14]}$. Massaging the injection site has been shown to enhance contrast uptake into the lymphatic system. The scan typically takes 20-50 min to obtain adequate imaging for upper limb studies and up to $1.5 \mathrm{~h}$ for the lower limb, during which the patient must remain still in the MRI machine $\mathrm{e}^{[3,8,15,23]}$ [Figure 1].

Gadolinium-enhanced T1-weighted sequences have been shown to provide higher spatial resolution, higher signal-to-noise ratio, and fewer artefacts than the non-contrast T2-weighted sequences. However, the range of visualised lymphatic pathways may be limited by the location of the injection site $^{[14]}$. Contrast-enhanced MRL appears to be optimal for evaluating distal lymphatic vessels, whereas non-contrast MRL may be superior for evaluating proximal and deeper lymphatic trunks ${ }^{[1,3,31]}$. Lohrmann et al.$^{[16]}$ reported that $92 \%$ of lymphatic vessels in the lower leg were visualised after contrast injection, whereas only $54 \%$ of lymphatic vessels in the upper leg in the same patients were seen. Several papers suggest that a combination of the two scans should be performed to provide clinicians with a complete assessment ${ }^{[2,6,7,14,17,31]}$.

\section{MR venogram}

Venous contamination during contrast-enhanced MRL poses difficulty in interpreting images, as some of the contrast administered subcutaneously will be absorbed into the venous system. Lohrmann et al. ${ }^{[1,26]}$ reported that $100 \%$ of patients studied had concomitant venous enhancement, and Notohamiprodjo et al. ${ }^{[2]}$ 


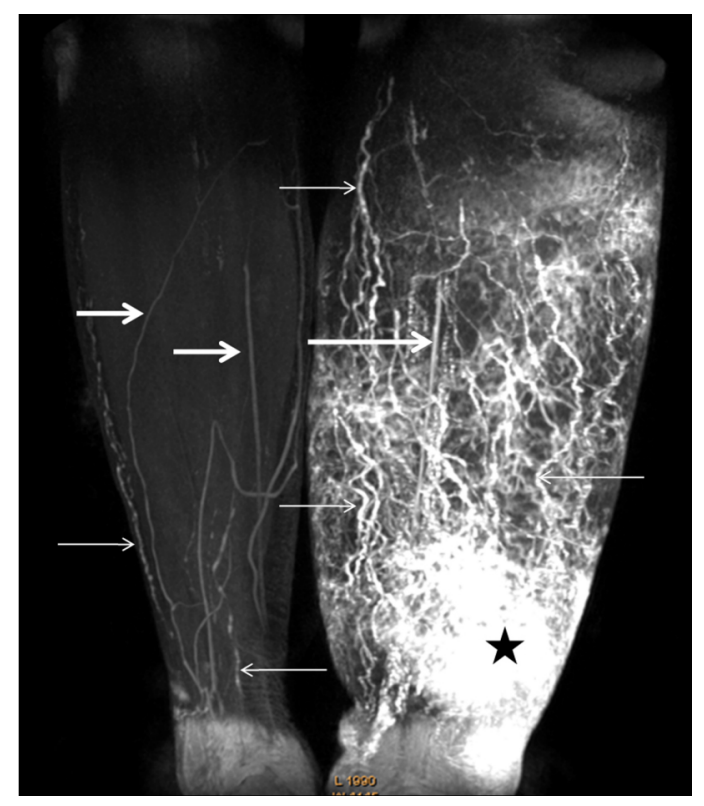

Figure 1. Contrast-enhanced MRL of a woman with stage III lymphoedema. The image shows numerous dilated lymph vessels in the left calf with a classical tortuous beaded appearance (small arrows), and unaffected lymphatic vessels in the right calf (small arrows). The star indicates an area of dermal backflow and the low signal intensity linear structures are veins (large arrows) ${ }^{[24]}$.

reported that 15 out of 16 patients (93.75\%) had venous enhancement. Therefore, a final scan with intravenous contrast injection can be performed, which is then visually compared to the first image to differentiate lymphatic vessels from veins ${ }^{[2,3]}$. This scan can be rendered not useful if there is a large degree of patient movement, which can frequently occur during the pauses between the phases ${ }^{[2]}$.

Mitsumori et al. ${ }^{[6]}$ used two techniques to overcome venous contamination; the incorporation of a delayed MR venogram using the same GBCA to locate the course of superficial veins and creating venous suppression by administering a small paramagnetic iron-oxide blood-pool contrast agent (ferumoxytol) intravenously to remove venous signal secondary to T2 subtraction [Figure 2]. Maki et al. ${ }^{[30]}$ adopted this second technique known as dual-agent relaxivity contrast (DARC) by giving intravenous ferumoxytol before or after the subcutaneous gadolinium injection and then lengthening the echo time (TE). Intravenous ferumoxytol remains exclusively intravenous and shortens the $\mathrm{T} 2^{*}$ in blood vessels, allowing $\mathrm{T} 2^{*}$-mediated subtraction of all vascular signals with lengthened TE so that only the gadolinium-containing lymphatic vessels are visualised. Gadolinium-enhancing lymphatics and blood vessels can both be seen by using short TE; therefore, a combination of a short- and long-TE pair is used in this technique to "turn on" and "turn off" the venous signal. Maki et al. ${ }^{[30]}$ suggest that this technique should be reserved for patients with significant venous contamination, which obstructs the scan interpretation, due to the FDA's warning that ferumoxytol can cause severe allergic reactions and needs to be administered with caution. Of note, the DARC technique is equally effective at both $1.5 \mathrm{~T}$ and $3 \mathrm{~T}$ field strengths, which may be useful to centres that do not have access to 3 T MRI machines.

\section{Non-contrast MRL}

Introduced in the early 1990s, T2-weighted 3D gradient-echo MRI sequence is a safe and non-invasive technique used to visualise slow-moving or stagnant fluid within the body, such as that within impaired lymphatic vessels ${ }^{[8,9,14,15,19,21,31]}$. It is performed with a long TR/TE and is the same technique used for MR cholangiopancreatography, MR urography, and MR myelography, producing a near-complete signal loss 

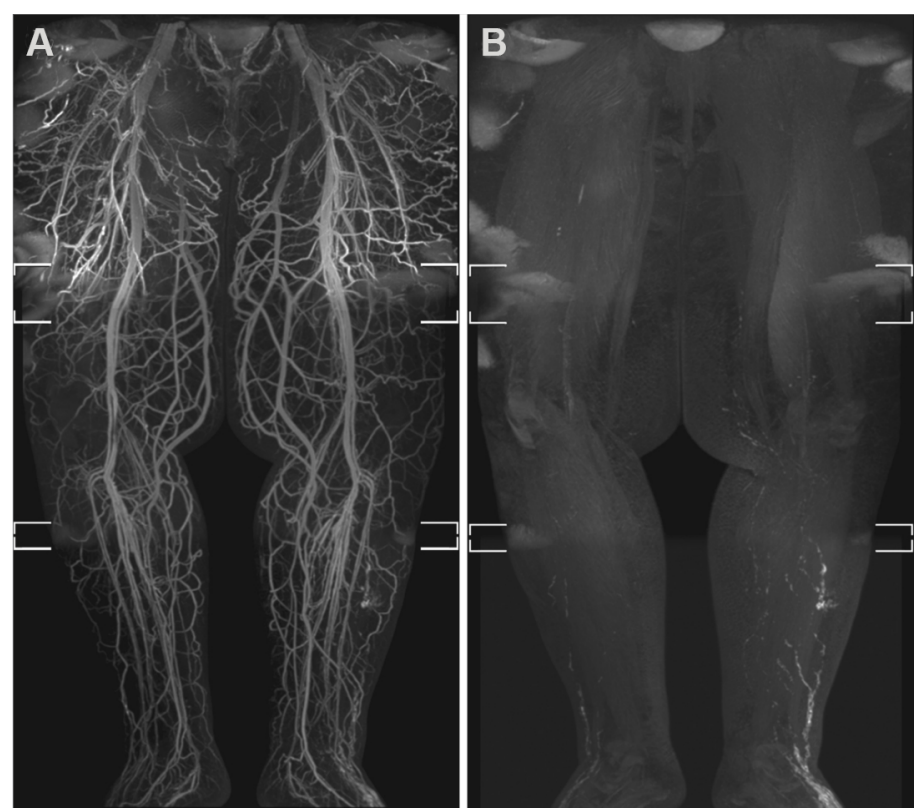

Figure 2. Venous signal suppression with ferumoxytol in a man with secondary lymphoedema. Bilateral lower limb MRL using ferumoxytol to suppress veins through its $\mathrm{T} 2^{*}$ shortening blood-pool effect. Extensive venous enhancement is seen in the Coronal fused MIP MRL (A), masking the lymphatic vessels in the left lateral lower leg. Coronal fused MIP after an increase in Dixon echo time to 5.8/6.9 ms (B) demonstrates venous signal suppression, allowing the lymphatic channels in the left lower leg to be seen more clearly ${ }^{[6]}$. MIP: Maximum intensity projection; MRL: magnetic resonance lymphography.

from background tissue, which displays static fluids as white on a black background without the need for contrast injection $^{[8,9,14,1,2,21]}$ [Figure 3]. This method provides anatomical detail of the lymphatic system, which is used to assess the severity and distribution of lymphoedema ${ }^{[2,7,31]}$. Non-contrast MRL with fat suppression has been reported to have a greater capability to identify the extent of tissue fluid accumulation and evaluate the severity of oedema than contrast-enhanced $\mathrm{MRL}^{[14]}$. In addition to showing dilated lymphatic vessels, skin thickening and pathological soft tissue changes, such as fat deposits and fibrotic tissue, can be observed in unenhanced $\mathrm{MRL}^{[7,13,25,28]}$. T2 fat-saturated sequences can also exclude lipoedema by identifying fluid stranding within the fat as the cause of fat hypertrophy ${ }^{[7,31]}$. Using heavily T2-weighted MRI with fat- and fluid-sensitive sequences, Borri et al. ${ }^{[32]}$ were able to quantify the volumes of fat and fluid within lymphoedematous limbs in order to target treatment. Patients with fluid-predominant lymphoedema benefit from compression therapy, whereas those found to have higher fat volumes should be considered for liposuction.

Compared to contrast-enhanced MRL, non-contrast MRL shows a greater number of lymphatic vessel ${ }^{[14]}$. The origin of each lymphatic vessel is independent, and injected contrast might reach only a part of the lymphatic vessels in limbs ${ }^{[33]}$. It was reported in one paper that the maximum diameter of selected vessels seen on unenhanced MRL was found to be larger than those on enhanced MRL, with the authors hypothesizing that this could be due to either the poorer spatial resolution allowing surrounding tissue oedema to be included in the vessel measurement or the limited contrast uptake in enhanced $\mathrm{MRL}^{[14]}$.

Although non-contrast MRL does not provide any functional information, the anatomical information provides useful pre-operatively for identifying sites for lymph node transplantation or suitable sites for lymphovenous anastomosis, along with mapping the lymphatic drainage ${ }^{[19]}$. Non-contrast MRL is a useful imaging modality for evaluating the extent and severity of lymphoedema, but cannot always identify a cause 

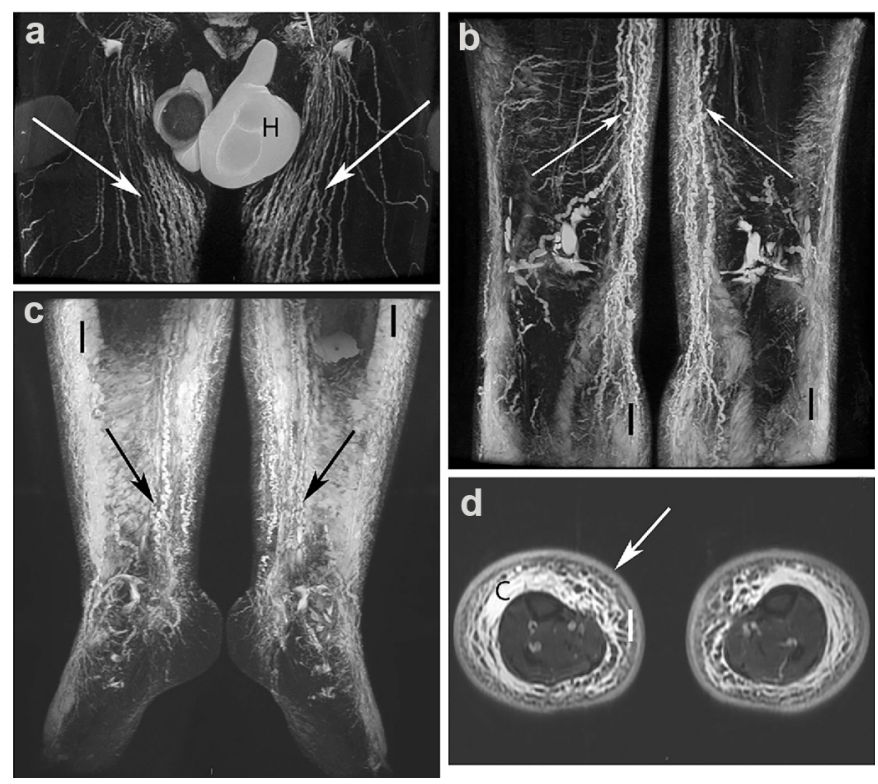

Figure 3. Non-contrast FSE MRL of a man with moderate hyperplasic bilateral lower limb oedema. (a) Increased number of dilated lymphatic iliac and inguinal trunks (arrows). Bilateral hydrocoele $(\mathrm{H})$ present. (b, c) Dilated lymphatic vessels (arrows) seen at the lower levels of the limbs and fluid infiltration (I) of subcutaneous fat. (d) Water IDEAL T2 FSE image. Bilateral fluid infiltration (I) of subcutaneous fat with a honeycomb pattern, moderate epifascial collection (C) and increased thickness of the dermis (arrow) ${ }^{[20]}$.

for the lymphoedema without combining it with contrast-enhanced scans ${ }^{[14]}$.

\section{INTERPRETATION OF MRL}

\section{Lymphatic vessels}

The first role of MRL is to define the severity and extent of lymphoedema, and the second is to identify the presence, number, course, and location of the lymphatic vessels ${ }^{[2,6]}$. Lymphatic vessels within limbs affected by lymphoedema can be identified by their tortuous, beaded, irregular, and dilated appearance with high signal intensity due to lymph stasis, as opposed to normal lymphatics, which are generally small, ill-defined, fewer in number. It is also worth noting that healthy lymphatics may not always be seen due to the faster flow of lymphatic fluid, which results in faster contrast washout in enhanced T1-weighted sequences and less signal intensity than the stagnant fluid in $\mathrm{T} 2$-weighted sequences ${ }^{[2-4,7,11,14,15,19,24,26]}$.

Collateral vessel formation and delayed enhancement of both lymphatic vessels and lymph nodes are also pathological features that can be observed ${ }^{[7]}$. Lymph leakage can be seen on contrast-enhanced MRL following trauma or iatrogenic damage to the vessel and in post-operative anastomotic leaks ${ }^{[3,4,7,17,29]}$. Postcontrast scans show a vast accumulation of contrast agents in affected limbs of patients with unilateral lymphoedema compared to their healthy limbs ${ }^{[15]}$.

Of note, there is some variation in the appearance of lymphatic vessels. The typical tortuous beaded appearance described above is seen in $80 \%-90 \%$ of lymphoedema patients, but lymphatic vessels may also appear rectilinear in a smaller cohort of patients ${ }^{[3,18]}$. In support of this figure, Liu et al ${ }^{[29]}$ found tortuous and significantly dilated lymphatic vessels in 104 out of 123 patients (84.5\%) with upper limb lymphoedema secondary to breast cancer therapy. It is also worth noting that primary lymphoedema can be categorised as either aplasia/hypoplasia or hyperplasia, which can account for the variation in appearance ${ }^{[13,25]}$. 


\section{Veins}

In contrast to lymphatic vessels, veins appear straighter, smooth-walled, have focal areas of bulging from the valves, and have lower signal intensity due to contrast washout-out from a faster flow. However, their diameter can be similar to that of affected lymphatic vessels ${ }^{[3,7,18,23,24,26]}$. The enhancement kinetics of lymphatic vessels and veins differs throughout dynamic contrast studies, with lymphatics becoming more enhanced proximally over time and venous enhancement decreasing over time ${ }^{[2]}$. Performing an MR venogram and comparing it to the MRL can be helpful in distinguishing the veins.

\section{Lymphatic vessel diameter}

Both Mazzei et al. ${ }^{[1]}$ and Baz et al. ${ }^{[18]}$ claim that lymphatic vessel diameter can be useful in assisting interpretation. However, lymph vessels dilation is only seen in $20 \%$ of lymphoedematous limbs, and both authors agree that this is not a reliable feature for identifying affected lymphatic vessels ${ }^{[13]}$. Vessel dilation in the lower limb is defined by White et al. ${ }^{[7]}$ as $>3 \mathrm{~mm}$ below the knee and $>5 \mathrm{~mm}$ above the knee. Bae et al. ${ }^{[24]}$ found the mean diameter of upper limb lymphatic vessels to be $1.98 \pm 0.30 \mathrm{~mm}$ in healthy individuals and $3.06 \pm 0.78 \mathrm{~mm}$ in individuals affected with lymphoedema, with the difference being statistically significant. Other reported diameter ranges in lymphoedematous limbs were 0.5-10 $\mathrm{mm}$ in lower limbs and 0.5-5 $\mathrm{mm}$ in upper limbs ${ }^{[4,27,29]}$. Lu et al. ${ }^{[14]}$ found a statistically significant discrepancy in the diameter of affected lower limb lymphatic vessels between contrast and non-contrast MRL, with the mean vessel diameter measuring $3.41 \pm 1.05 \mathrm{~mm}$ on contrast-enhanced MRL and $4.28 \pm 1.53 \mathrm{~mm}$ on non-contrast MRL. They hypothesized that this was due to the high endolymphatic pressure within the vessels that affect the contrast uptake.

\section{Dermal backflow}

Dermal backflow is the dispersion of contrast into the dermal lymphatics or surrounding soft tissue, suggesting obstruction of the lymphatic vessel, and identifies areas of high intralymphatic pressure and excess lymphatic fluid ${ }^{[2,18,23]}$. It typically appears around $20 \mathrm{~min}$ after contrast injection and becomes more prominent over time ${ }^{[7]}$. Dermal backflow appears as an irregular, patchy, high signal intensity area, and the reported prevalence is similar across multiple papers; $46.7 \%$ of patients with primary and secondary lymphoedema, 53.84\% of patients with primary lower limb lymphoedema, 63\% of patients with secondary lower limb oedema, and 52\% of patients with oncology-related lower limb lymphoedema ${ }^{[3,16,18,23]}$.

\section{Honeycombing}

A honeycomb appearance (honeycombing) is the term used to describe the infiltration of lymph fluid into subcutaneous fat or soft tissue, which is best appreciated on non-contrast heavily T2-weighted sequences but can also be identified on contrast-enhanced $\mathrm{MRL}^{[7,14,17,21]}$. It has also been hypothesized that it could be due to the opacification of multiple tiny peripheral lymphatic vessels associated with lymphatic backflow ${ }^{[14]}$. Honeycombing appears as a trabecular structure with enlarged fat pockets surrounded by lines of fluid or fibrous tissue, and is characteristics of lymphoedema differentiating it from venous oedema, lipoedema, and morbid obesity by demonstrating a combination of both fat deposition with fluid accumulation ${ }^{[8,19-21]}$. Two studies independently found honeycombing to be present in $47 \%$ of subjects with known lymphoedema ${ }^{[3,14]}$.

\section{Lymph nodes}

MRL can be used to characterise the size, border, architecture, fluid transport, and the number of lymph nodes, yet the appearance of lymph nodes on MRL is markedly variable due to the varying pathologies ${ }^{[34]}$. Secondary lymphoedema caused by surgery and/or radiation shows smaller and fewer lymph nodes in the affected limb, whereas lymphoedema caused by lymph node metastasis has been found to demonstrate enlarged lymph nodes ${ }^{[25,31]}$. Homogenous signal loss in the lymph nodes can be due to either complete fibrosis of the nodes or, by contrast, not reaching the nodes because of stagnant flow upstream in the pre- 
nodal lymphatic vessels ${ }^{[15]}$. Lymph nodes in primary lymphoedematous limbs have also been shown to have reduced peak enhancement and slower washout, indicating abnormal function ${ }^{[13]}$. One paper documented a variety of lymph node appearances and categorized them according to three major categories; aplasia/hypoplasia, hyperplasia, or structural abnormalities ${ }^{[27]}$.

Normal lymph nodes may appear as a converging plexus of lymphatic vessels or have a large fatty component in the centre ${ }^{[21]}$. Another study found that normal inguinal lymph nodes have a consistent appearance of being spherical or oval and measure approximately $1 \mathrm{~cm}$ in diameter, whilst the lymph nodes in primary lower limb lymphoedema tend to have greater variation in outline, number, and volume ${ }^{[15]}$.

\section{ROLE IN DIAGNOSIS AND STAGING}

Lymphoedema diagnosis is based on history and clinical assessment, and the most popular staging system used is the International Society of Lymphology (ISL) system. Stages range from 0/Ia (subclinical) to III, based on the degree of oedema and fibrotic soft tissue changes and the change in oedema on limb elevation [Table 1$]^{[1,12,28]}$. Early diagnosis and management can prevent chronic lymphoedema, and it is suggested that those at risk of developing lymphoedema (i.e., post-operative or those receiving cancer treatment) should commence physiotherapy in stage $0 /$ Ia to prevent the progression of the disease ${ }^{[35]}$. It has been found that MRL can be helpful in both diagnosing and staging lymphoedema and can identify impaired or abnormal lymphatic drainage patterns before any clinical signs are evident ${ }^{[2,14]}$. This makes it a valuable tool in early detection and, therefore, early management of the disease. There is a strong correlation between the severity of lymphoedema found on MRL with the clinical staging of the disease, both in primary and secondary lymphoedema ${ }^{[19,23]}$. Comparison of the affected limb to the contralateral unaffected limb can also assist both with diagnosis and establishing the extent of disease.

Due to its high spatial resolution, MRL is capable of visualizing lymphatic vessels and lymph nodes with precise anatomical detail in lymphoedematous limbs. It allows clinicians to view lymph transport and identify the cause of obstruction to the lymphatic system or any anatomical abnormalities that may be the cause of primary lymphoedema ${ }^{[8,13,15,36]}$.

Enhanced MRL has also proven useful for the diagnosis and staging of malignant lymph nodes ${ }^{[15]}$. Flow velocities on dynamic contrast-enhanced studies can provide a means of quantitatively grading impaired lymphatic transport ${ }^{[2]}$. This diagnostic tool involves calculating the speed of the lymphatic fluid in affected vessels by measuring the distance contrast travels between each time sequence, which correlates with the transport capabilities of the lymphatic vessels ${ }^{[13,15,25]}$.

Other MRI sequences (for example, T2 STIR) can detect extra-lymphatic changes, such as the composition of subcutaneous tissue, differentiating predominantly fluid accumulation in the early stages of the disease from the later stages that predominantly have adipose and fibrosclerotic tissue deposition ${ }^{[2]}$. As well as indicating the type of subcutaneous fluid present, it also provides information on the location and volume of this extracellular fluid. Differentiating these stages is important not only in staging the disease, but also in determining which surgical procedure is suitable to manage it ${ }^{[2,11]}$.

In a study by Mihara et al. ${ }^{[36]}$, all 21 patients with known stage 1 lymphoedema were positively identified using MRI, However, only 13 showed positive findings with lymphoscintigraphy for qualitative assessment, giving a higher sensitivity for the diagnosis of lymphoedema in the early stages of the disease using MRL (sens $=1$ ) than with using lymphoscintigraphy (sens $=0.62$ ). In addition to this study, non-contrast MRL has successfully been able to classify primary lymphoedema into its main pathological groups; hyperplasic, 
Table 1. Comparing lymphoedema stages with clinical signs and radiological signs ${ }^{[6,11,22]}$

\begin{tabular}{|c|c|c|}
\hline Stage (ISL) & Clinical signs \& symptoms & Radiological signs \\
\hline $\begin{array}{l}\text { 0/la } \\
\text { (subclinical) }\end{array}$ & $\begin{array}{l}\text { - Swelling is not evident, despite impaired lymph transport } \\
\text { - Asymptomatic or subjective symptoms of limb heaviness } \\
\text { - No clinical signs }\end{array}$ & $\begin{array}{l}\text { - Subtle changes in subcutaneous tissue fluid composition } \\
\text { - Abnormal lymphatic vessels } \\
\text { - Fewer lymph nodes than lymphatic vessels } \\
\text { - Interruption of lymphatic vessels with or without distal } \\
\text { lymphatic vessel dilatation }\end{array}$ \\
\hline I (mild) & $\begin{array}{l}\text { - Early accumulation of protein-rich fluid } \\
\text { - May have pitting oedema (without fibrosis) } \\
\text { - Oedema subsides with limb elevation within } 24 \mathrm{~h}\end{array}$ & $\begin{array}{l}\text { - Less visualized lymphatic vessels } \\
\text { - Delay of contrast agent transport }\end{array}$ \\
\hline II (moderate) & $\begin{array}{l}\text { - Pitting oedema (may no longer pit as the fibrosis progresses, } \\
\text { reducing tissue compliance) } \\
\text { - Oedema does not resolve with limb elevation alone } \\
\text { - Loss of joint flexibility }\end{array}$ & $\begin{array}{l}\text { - Honeycomb pattern } \\
\text { - Contrast agent accumulation between fat surrounded by } \\
\text { fibrotic tissue (late stage } 2 \text { ) }\end{array}$ \\
\hline III (severe) & $\begin{array}{l}\text { - Lymphostatic elephantiasis } \\
\text { - Pitting usually absent } \\
\text { - Skin hyperkeratosis/acanthosis } \\
\text { - Fat deposits } \\
\text { - Fibrosis } \\
\text { - Warty overgrowths } \\
\text { - Marked functional loss }\end{array}$ & $\begin{array}{l}\text { - Large, irregular, patchy shaped dermal backflow } \\
\text { - Increased subcutaneous thickness and diffuse fibrosis } \\
\text { - Dilated lymph vessels and damaged lymph vessels even } \\
\text { with severe fibrosis } \\
\text { - Tortuous and deep-seated lymph vessels (due to } \\
\text { overgrowth of adipose tissue) }\end{array}$ \\
\hline
\end{tabular}

ISL: International Society of Lymphology.

aplasic, hypoplasic and normal patterns ${ }^{[12,19,21]}$.

\section{ROLE IN SURGICAL PLANNING}

MRL plays a vital role in planning surgeries and choosing the appropriate procedure based on the structural abnormality causing the lymphoedema. Lymph node transfer (LNT) is used for lymphoedema of moderate severity and in patients who have had lymph node dissections or radiotherapy as part of their oncology treatment. LNT utilizes functioning vascularized lymph nodes, which can be harvested either with/without a skin paddle and are then re-anastomosed with the blood vessels within the lymphoedematous limb ${ }^{[37,38]}$. This technique not only improves lymphoedema but has a significant effect on reducing cellulitis in limbs. Lin et al..$^{[38]}$ used lymphoscintigraphy for both pre-operative planning and post-operative follow-up in patients following LNT using tissue flaps, which identified increased uptake of the radio-labelled tracer and reduced lymph stasis post-operatively. Although this study used lymphoscintigraphy, in theory, the same method could be applied using MRL could have the potential to replace lymphoscintigraphy to assess lymphatic function both before and after LNT surgery.

LVA is the surgical technique to create a peripheral shunt between lymphatic vessels and veins in early lymphoedema ${ }^{[5,19]}$. The first step in planning for LVA surgery is to identify the lymphatic channels and venules to anastomose ${ }^{[2,28,30]}$. For anastomosis, both lymphatic vessels and their adjacent venules must be between $0.3-0.8 \mathrm{~mm}$ in calibre, and the lymphatics must not be sclerotic or tortuous ${ }^{[5,10,1,23]}$. The number, calibre, anatomical location, and distribution of the lymphatic vessels in the affected limb should be evaluated prior to surgery to ensure that they fit the criteria for surgery and predict the chance of a successful outcome $e^{[2,3,1,23]}$. Zeltzer et al. ${ }^{[28]}$ successfully used contrast-enhanced MRL to identify functional lymphatic channels that were in close proximity to adjacent veins with a matching calibre, and within a region of fluid accumulation, which they were then able to mark as the optimal site for LVA surgery. They also identified fat hypertrophy in a number of patients who then went on to have targeted liposuction either as an adjunct to LVA or as a single therapy. Liposuction shows promising long-term results and is suitable for those with at least stage II lymphoedema, in which adipose hypertrophy and fibrosis are present, which cannot be removed by compression or surgical diversion of lymph fluid alone $e^{[39,40]}$. 
Lu et al. ${ }^{[17]}$ found that contrast-enhanced MRL identified the location of post-traumatic inguinal lymphatic vessel leakage in $93.8 \%$ of patients studied, all of whom were reported to have successful surgical ligation of the damaged vessel once it had been located. On conventional T2 MRI, these regions of high signal intensity cannot be distinguished from other fluid-filled lesions, and therefore cannot be accurately confirmed as the site of lymphatic vessel leakage.

MRL is useful in both identifying the condition of lymphatic vessels and assessing the patient's response to surgery by measuring the reduction in oedema, limb size, and dermal backflow ${ }^{[2,3,8,18,23]}$. Arrivé et al ${ }^{[20]}$ found that non-contrast MRL may be useful as an objective technique to analyse the post-operative results of lymph node transplantation.

\section{BENEFITS AND LIMITATIONS \\ Benefits}

Not only is MRL widely recognised for having a higher spatial and temporal resolution than lymphoscintigraphy, but it has many other benefits that contribute to its increasing popularity as an imaging modality for the lymphatic system ${ }^{[2,7,1,1,18,21,24,25,30]}$. MRL utilises contrast uptake into lymphatic vessels, transportation through the lymphatic system, and nodal uptake to give both high-quality anatomical and functional assessments of the lymphatic drainage system in one process ${ }^{[2,3,7,71,15,18,19,23,25,27,34]}$. It gives clinicians the option of using one single scan for the diagnosis, surgical planning, and treatment evaluation for patients with lymphoedema.

MRL is consistently acknowledged for being quick, minimally invasive and avoiding ionizing radiation $^{[2,3,1,1,1,8,21,23,25,26]}$. GBCAs were considered safe, and no reactions or short-term complications in patients with normal renal function were documented in any of the papers reviewed. In addition to being safe, it has a reasonable specificity of absorption and transport within the lymphatic system ${ }^{[15]}$.

The T2-weighted fat-suppressed MRI sequences provide information on other soft tissue changes, such as adipose deposition and fibrosis ${ }^{[2,13,25]}$, and differentiate from other types of peripheral oedema ${ }^{[1,25]}$. MRIs provide a wider field of view than lymphoscintigraphy, and by creating $3 \mathrm{D}$ images, superimposed vessels are less likely to obscure detail ${ }^{[34]}$. Since MRL does not require the cannulation of a single lymphatic vessel, more vessels have the potential to uptake the contrast and be visualised. The detection rates of lymphatic vessels and lymph nodes have been found superior in MRL compared to lymphoscintigraphy ${ }^{[13,24,25]}$. MRL not only provides a real-time assessment of the transport function of the lymphatic system, but it can be performed in a reasonable timeframe ${ }^{[15]}$.

MRL can detect subclinical lymphoedema before it progresses to severe limb enlargement in the later stages, allowing for conservative management to be commenced earlier and prevent progression to advanced disease $^{[2,36]}$. It is also valuable in a post-operative setting to monitor response to treatment and early complications. Circumferential measurements of limbs at fixed positions are common practice for lymphoedema surveillance. However, MRL is a more accurate form of surveillance ${ }^{[20]}$. MRL has a range of alternative uses, including tumour staging, evaluating retroperitoneal and abdominal lymphatic vessels, visualising the thoracic duct, and it can be used to investigate post-operative lymphocele formation ${ }^{[7,8]}$. Bioimpedance spectroscopy (BIS) also demonstrated positive interim results to prevent breast cancerrelated lymphoedema compared to tape measurement ${ }^{[4]}$. The combination of BIS and MRL has the potential to help increase our understanding of the pathophysiology of lymphoedema and is a useful tool for ongoing research. 


\section{Limitations}

Despite its many advantages, MRL does pose some challenges, particularly in relation to its use of gadolinium-based contrast. It should be noted that gadolinium has been shown to store within the body as an inert substance bound to molecules, and the long-term effects are yet to be confirmed ${ }^{[42,43]}$. Numerous studies have observed retention of gadolinium in the brain (specifically deep nuclei), bones, and skin following GBCA use ${ }^{[42-44]}$. Furthermore, many contrast MRL studies report the use of gadobenate dimeglumine for the contrast medium, which has a linear chemical structure ${ }^{[1-4,6,6,13,14,16-18,22,23,25,27-30]}$. Linear GBCAs are reported to result in greater gadolinium retention for a longer time period than macrocyclic GBCAs, likely relating to the chemical stabilities of these agents ${ }^{[4,45]}$. Although the health implications of gadolinium retention are not known, the International Society of Magnetic Resonance in Medicine Safety Committee recommends that the use of GBCAs are avoided when not necessary ${ }^{[44]}$. Additionally, the FDA has issued warnings relating to the retention of GBCAs and recommends that repeat use of GBCA is minimised where possible ${ }^{[46]}$. At this stage, as there is no strong evidence regarding the side effects of GBCAs, the United States FDA and Australian TGA have not removed any agents from the market, whilst the European Medicines Agency has removed the linear chelates gadodiamide, and gadopentetate from the market ${ }^{[44]}$. In current practice, linear GBCAs are rarely used and it should be noted that it is now common practise to use macrocyclic agents for contrast-enhanced MRIs.

Due to its water solubility, gadolinium contrast is taken up into the venous system. It does not occur with the colloid-binding tracers used in lymphoscintigraphy, which are more specific for the lymphatic system than gadolinium ${ }^{[3,22,24]}$. Enhancement of the venous system may complicate analysis since it can be difficult to distinguish veins from lymphatic vessels $s^{[3,6,8,8,98,1,9,22,30]}$. Additionally, unaffected limbs do not image well on both enhanced and non-enhanced MRL owing to their faster lymphatic flow resulting in contrast washout on enhanced T1-weighted scans and less signal intensity on T2-weighted scans (as signal intensity is caused by static fluid) $)^{[19,20]}$. It limits the understanding of the location of healthy vessels in the affected limb, and therefore it may be difficult to identify potential collateral drainage pathways. Furthermore, lymphatic vessels must have a degree of function to uptake contrast. Several studies identified patients with clinical lymphoedema that had completely non-functional lymphatics with no contrast uptake on MRL, most commonly seen in aplasia/hypoplasia primary lymphoedema ${ }^{[3,4,13,16,27-29]}$. It has also been recognised in two articles that the larger proximal lymphatic trunks do not enhance as well as the smaller distal vessels on contrast-enhanced MRL likely also from contrast washout ${ }^{[8,9]}$. There are a variety of protocols regarding the exact location and number of sites for contrast injection, and the validity of these protocols has not been compared to form a standard protocol for optimal anatomical detail ${ }^{[34]}$.

As with all injections, there is a small risk of hypersensitivity to the contrast agent, injection site infection, pain at the injection site, intra-vascular contrast administration, and pulmonary embolism. Some papers excluded patients with renal insufficiency from their study cohort. However, there is insufficient evidence on the effect that gadolinium-based contrasts have on renal function ${ }^{[11,14,16,17,18,22-24,26]}$. Nephrogenic systemic fibrosis has been reported as a complication of GBCA exposure in patients with severe renal failure. However, these reports are not specific to $\mathrm{MRL}^{[41-43]}$. Of note, it has s been found that only $3 \%$ of patients with end-stage renal failure developed nephrogenic systemic fibrosis following the administration of $\mathrm{GBCAs}^{[42]}$. However, a review article of MRL described that no adverse events or allergic reactions were noticed in primary and secondary lymphedema patients ${ }^{[47]}$.

Contraindications for MRL are the same as for other types of MRI, including patients with severe claustrophobia, pacemakers, ferromagnetic intracerebral clips, or any other electrically, magnetically, or mechanically activated implants ${ }^{[2,24,25]}$. Some patients may have difficulty remaining still within the confined space of an MRI machine due to discomfort, anxiety, chronic back pain, and movement disorders ${ }^{[3]}$. Patient 
cooperation is necessary to obtain good quality and useful scans.

No studies were found to report a cost analysis of MRL compared to other imaging modalities for lymphoedema. It would be a future area of interest for determining the practicalities of using MRL and the cost versus benefit of MRL compared to lymphoscintigraphy and indocyanine green lymphography.

\section{CONCLUSION}

MRL is recognised as an accurate and safe imaging modality to visualize the lymphatic pathways. MRL can detect secondary changes in the skin and subcutaneous tissue, accurately demonstrate the extent and location of oedema, and provide a functional assessment of the lymphatic pathways. It is a valuable tool in diagnosing, classifying, staging, and identifying the cause of both primary and secondary lymphoedema. In addition, it has been reported useful for pre-operative planning and post-operative surveillance.

Both contrast and non-contrast MRL scans have their strengths and weaknesses, and obtaining a combination of both heavily $\mathrm{T} 2$-weighted and post-contrast T1-weighted sequences is recommended by many articles as the optimal method for assessing lymphoedema. As MRL becomes increasingly used, we will likely learn the extent of its benefits and limitations and may potentially use it to enhance our knowledge of lymphoedema further.

\section{DECLARATIONS}

\section{Authors' contributions}

Made substantial contributions to conception and design of the study and performed data analysis and interpretation: Calderwood A, Ho-Shon K, Suami H

Performed data acquisition, as well as provided editorial support: Thompson B

\section{Availability of data and materials}

Not applicable.

\section{Financial support and sponsorship}

None.

\section{Conflicts of interest}

All authors declared that there are no conflicts of interest.

\section{Ethical approval and consent to participate}

Not applicable.

\section{Consent for publication}

All figures were obtained copyright permission for use in this manuscript.

\section{Copyright}

(c) The Author(s) 2021.

\section{REFERENCES}

1. Mazzei FG, Gentili F, Guerrini S, et al. MR lymphangiography: a practical guide to perform it and a brief review of the literature from a technical point of view. Biomed Res Int 2017;2017:2598358. DOI PubMed PMC

2. Mitsumori LM, McDonald ES, Wilson GJ, Neligan PC, Minoshima S, Maki JH. MR lymphangiography: how I do it. J Magn Reson Imaging 2015;42:1465-77. DOI

3. Mazzei MA, Gentili F, Mazzei FG, et al. High-resolution MR lymphangiography for planning lymphaticovenous anastomosis treatment: a single-centre experience. Radiol Med 2017;122:918-27. DOI PubMed 
4. Liu NF, Yan ZX, Wu XF, Luo Y. Magnetic resonance lymphography demonstrates spontaneous lymphatic disruption and regeneration in obstructive lymphedema. Lymphology 2013;46:56-63. PubMed

5. Chang DW, Suami H, Skoracki R. A prospective analysis of 100 consecutive lymphovenous bypass cases for treatment of extremity lymphedema. Plast Reconstr Surg 2013;132:1305-14. DOI PubMed

6. Mitsumori LM, McDonald ES, Neligan PC, Maki JH. Peripheral magnetic resonance lymphangiography: techniques and applications. Tech Vasc Interv Radiol 2016;19:262-72. DOI PubMed

7. White RD, Weir-McCall JR, Budak MJ, Waugh SA, Munnoch DA, Sudarshan TA. Contrast-enhanced magnetic resonance lymphography in the assessment of lower limb lymphoedema. Clin Radiol 2014;69:e435-44. DOI PubMed

8. Cellina M, Oliva G, Menozzi A, Soresina M, Martinenghi C, Gibelli D. Non-contrast magnetic resonance lymphangiography: an emerging technique for the study of lymphedema. Clin Imaging 2019;53:126-33. DOI PubMed

9. Cellina M, Gibelli D, Floridi C, Oliva G. Volumetric analysis of non-contrast magnetic resonance lymphangiography in patients affected by lower extremities primary lymphedema. Radiol Med 2020;125:432-5. DOI PubMed

10. Nagase T, Gonda K, Inoue K, et al. Treatment of lymphedema with lymphaticovenular anastomoses. Int J Clin Oncol 2005;10:304-10. DOI PubMed

11. Long X, Zhang J, Zhang D, et al. Microsurgery guided by sequential preoperative lymphography using ${ }^{68}$ Ga-NEB PET and MRI in patients with lower-limb lymphedema. Eur J Nucl Med Mol Imaging 2017;44:1501-10. DOI PubMed PMC

12. Linnitt N. Lymphoedema: recognition, assessment and management. Br J Community Nurs 2005;10:S20-6. DOI PubMed

13. Liu NF, Lu Q, Liu PA, Wu XF, Wang BS. Comparison of radionuclide lymphoscintigraphy and dynamic magnetic resonance lymphangiography for investigating extremity lymphoedema. Br J Surg 2010;97:359-65. DOI PubMed

14. Lu Q, Xu J, Liu N. Chronic lower extremity lymphedema: a comparative study of high-resolution interstitial MR lymphangiography and heavily T2-weighted MRI. Eur J Radiol 2010;73:365-73. DOI PubMed

15. Liu NF, Lu Q, Jiang ZH, Wang CG, Zhou JG. Anatomic and functional evaluation of the lymphatics and lymph nodes in diagnosis of lymphatic circulation disorders with contrast magnetic resonance lymphangiography. J Vasc Surg 2009;49:980-7. DOI PubMed

16. Lohrmann C, Földi E, Bartholomä JP, Langer M. MR imaging of the lymphatic system: distribution and contrast enhancement of gadodiamide after intradermal injection. Lymphology 2006;39:156-63. PubMed

17. Lu Q, Bui D, Liu NF, Xu JR, Zhao XH, Zhang XF. Magnetic resonance lymphography at 3T: a promising noninvasive approach to characterise inguinal lymphatic vessel leakage. Eur J Vasc Endovasc Surg 2012;43:106-11. DOI PubMed

18. Baz AA, Hassan TA, Atta A, El kholy MS. Role of contrast enhanced MRI lymphangiography in evaluation of lower extremity lymphatic vessels for patients with primary lymphedema. The Egyptian Journal of Radiology and Nuclear Medicine 2018;49:776-81. DOI

19. Arrivé L, Derhy S, Dahan B, et al. Primary lower limb lymphoedema: classification with non-contrast MR lymphography. Eur Radiol 2018;28:291-300. DOI PubMed

20. Arrivé L, Derhy S, Dlimi C, El Mouhadi S, Monnier-Cholley L, Becker C. Noncontrast magnetic resonance lymphography for evaluation of lymph node transfer for secondary upper limb lymphedema. Plast Reconstr Surg 2017;140:806e-11e. DOI PubMed

21. Arrivé L, Derhy S, El Mouhadi S, Monnier-Cholley L, Menu Y, Becker C. Noncontrast magnetic resonance lymphography. J Reconstr Microsurg 2016;32:80-6. DOI PubMed

22. Notohamiprodjo M, Baumeister RG, Jakobs TF, et al. MR-lymphangiography at 3.0 T--a feasibility study. Eur Radiol 2009;19:2771-8. DOI PubMed

23. Lu Q, Delproposto Z, Hu A, et al. MR lymphography of lymphatic vessels in lower extremity with gynecologic oncology-related lymphedema. PLoS One 2012;7:e50319. DOI PubMed PMC

24. Bae JS, Yoo RE, Choi SH, et al. Evaluation of lymphedema in upper extremities by MR lymphangiography: Comparison with lymphoscintigraphy. Magn Reson Imaging 2018;49:63-70. DOI PubMed

25. Liu N, Zhang Y. Magnetic Resonance lymphangiography for the study of lymphatic System in lymphedema. J Reconstr Microsurg 2016;32:66-71. DOI PubMed

26. Lohrmann C, Foeldi E, Speck O, Langer M. High-resolution MR lymphangiography in patients with primary and secondary lymphedema. AJR Am J Roentgenol 2006;187:556-61. DOI PubMed

27. Liu NF, Yan ZX, Wu XF. Classification of lymphatic-system malformations in primary lymphoedema based on MR lymphangiography. Eur J Vasc Endovasc Surg 2012;44:345-9. DOI PubMed

28. Zeltzer AA, Brussaard C, Koning M, et al. MR lymphography in patients with upper limb lymphedema: The GPS for feasibility and surgical planning for lympho-venous bypass. J Surg Oncol 2018;118:407-15. DOI PubMed

29. Liu NF, Wang BS. Functional lymphatic collectors in breast cancer-related lymphedema arm. Lymphat Res Biol 2014;12:232-7. DOI PubMed PMC

30. Maki JH, Neligan PC, Briller N, Mitsumori LM, Wilson GJ. Dark blood magnetic resonance lymphangiography using dual-agent relaxivity contrast (DARC-MRL): a novel method combining gadolinium and iron contrast agents. Curr Probl Diagn Radiol 2016;45:174-9. DOI PubMed

31. Liu N, Wang C, Sun M. Noncontrast three-dimensional magnetic resonance imaging vs lymphoscintigraphy in the evaluation of lymph circulation disorders: a comparative study. J Vasc Surg 2005;41:69-75. DOI PubMed

32. Borri M, Gordon KD, Hughes JC, et al. Magnetic resonance imaging-based assessment of breast cancer-related lymphoedema tissue composition. Invest Radiol 2017;52:554-61. DOI PubMed PMC

33. Shinaoka A, Koshimune S, Yamada K, et al. Correlations between tracer injection sites and lymphatic pathways in the leg: a nearinfrared fluorescence lymphography study. Plast Reconstr Surg 2019;144:634-42. DOI PubMed 
34. Clough RE. Magnetic resonance imaging to guide surgical practice. Eur J Vasc Endovasc Surg 2012;43:112-3. DOI PubMed

35. Torres Lacomba M, Yuste Sánchez MJ, Zapico Goñi A, et al. Effectiveness of early physiotherapy to prevent lymphoedema after surgery for breast cancer: randomised, single blinded, clinical trial. BMJ 2010;340:b5396. DOI PubMed PMC

36. Mihara M, Hara H, Araki J, et al. Indocyanine green (ICG) lymphography is superior to lymphoscintigraphy for diagnostic imaging of early lymphedema of the upper limbs. PLoS One 2012;7:e38182. DOI PubMed PMC

37. Becker C, Assouad J, Riquet M, Hidden G. Postmastectomy lymphedema: long-term results following microsurgical lymph node transplantation. Ann Surg 2006;243:313-5. DOI PubMed PMC

38. Lin $\mathrm{CH}$, Ali R, Chen SC, et al. Vascularized groin lymph node transfer using the wrist as a recipient site for management of postmastectomy upper extremity lymphedema. Plast Reconstr Surg 2009;123:1265-75. DOI PubMed

39. Brorson H. Liposuction gives complete reduction of chronic large arm lymphedema after breast cancer. Acta Oncol 2000;39:407-20. DOI PubMed

40. Brorson H, Svensson H. Liposuction combined with controlled compression therapy reduces arm lymphedema more effectively than controlled compression therapy alone. Plast Reconstr Surg 1998;102:1058-67; discussion 1068. PubMed

41. Ridner SH, Dietrich MS, Cowher MS, et al. A randomized trial evaluating bioimpedance spectroscopy versus tape measurement for the prevention of lymphedema following treatment for breast cancer: interim analysis. Ann Surg Oncol 2019;26:3250-9. DOI PubMed $\mathrm{PMC}$

42. Semelka RC, Ramalho M, Jay M. Summary of special issue on gadolinium bioeffects and toxicity with a look to the future. Magn Reson Imaging 2016;34:1399-401. DOI PubMed

43. Guideline on the use of gadolinium-containing MRI contrast agents in patient with renal impairment, Version 3. 2019, The Royal Australian and New Zealand College of Radiologists. Available from: https:/www.ranzcr.com/documents/553-revised-collegeguidelines-for-gadolinium-containing-mri-contrast-agents/file. [Last accessed on 5 Aug 2021].

44. Gulani V, Calamante F, Shellock FG, Kanal E, Reeder SB. Gadolinium deposition in the brain: summary of evidence and recommendations. Lancet Neurol 2017;16:564-70. DOI PubMed

45. Murata N, Murata K, Gonzalez-Cuyar LF, Maravilla KR. Gadolinium tissue deposition in brain and bone. Magn Reson Imaging 2016;34:1359-65. DOI PubMed

46. FDA Drug Safety Communication: FDA warns that gadolinium-based contrast agents (GBCAs) are retained in the body; requires new class warnings. Available from: https://www.fda.gov/drugs/drug-safety-and-availability/fda-drug-safety-communication-fda-warnsgadolinium-based-contrast-agents-gbcas-are-retained-body. [Last accessed on 5 Aug 2021].

47. Miseré RML, Wolfs JAGN, Lobbes MBI, van der Hulst RRWJ, Qiu SS. A systematic review of magnetic resonance lymphography for the evaluation of peripheral lymphedema. J Vasc Surg Venous Lymphat Disord 2020;8:882-92.e2. DOI PubMed 